\title{
Major clinical advances of depression: now and future
}

\author{
Peixin $\mathrm{He}^{1, \dagger}$, Xinyi $\mathrm{Liu}^{2, \dagger}$, Jiaqi Wen ${ }^{3, \dagger}$, Ying Zhang ${ }^{4, *, \dagger}$ \\ ${ }^{1}$ The Bolles School,7400 San Jose Blvd, Jacksonville, FL 32217, United States. \\ ${ }^{2}$ Long Island Lutheran Middle \& High school, Glen Head, NY, 11545. \\ ${ }^{3}$ Saint Anthony's High School, South Huntington, NY, 11747, United States. \\ ${ }^{4}$ Shen Wai International School, Nanshan, Shenzhen, 518053, China. \\ ${ }^{\dagger}$ These authors contributed equally to this work.
}

\begin{abstract}
Depression, as one of the largest influence disorders, needs more attention on its growing patients. Medicine treatment has been seeing as the major treatment for depression. As one of the most commonly used medicine, Selective Serotonin Reuptake Inhibitors (SSRIS) increases serotonin levels to treat depression effectively, and the data from clinical data prove its value. Furthermore, Serotonin and Norepinephrine Reuptake Inhibitors (SNRIs) and Monoamine Oxidase Inhibitors (MAOIs) treat depression by affecting at least one neurotransmitter, generally is chemical in the brain and digestive system. Other therapies were mostly as an auxiliary, such as psychological therapy, Electroconvulsive Therapy (ECT), Transcranial Magnetic Stimulation (TMS), and Vagus Nerve Stimulation (VNS). These therapies often work effectively by stimulating neurotransmitters' activity and increasing the activeness of the reward system. After new study and technology production, new development like ketamine can satisfy Individual Differences by more detailed inspection. Optogenetics in auxiliary treatment can be another way to increase the exactness of ECT and VNS after solving problems such as actual circuits in the whole network. In this review, we first focus mostly on drug treatments. We then summarize current information for other treatments and discuss the mechanisms that lead to in effect. Ultimately, we predict future development in the drug and optogenetics area. The previous study on depression treatment leads us a way to discover a future solution.
\end{abstract}

\section{Introduction}

Depression, as the most common life-threatening mental disorder, is a state of low mood and aversion to activity [1]. It can affect a person's thoughts, behavior, motivation, feelings, and well-being [2]. According to a report published by the World Health Organization, the number of people with this mental illness is between 2 and 6 percent worldwide. At the same time, depression is a leading cause of disability globally, affecting an estimated 300 million people worldwide, mostly women, young people, and the elderly. Doctors often spend a lot of time identifying and treating patients with various types of depression. About half of patients with clinical depression may not be recognized by their doctors at their first visit [3].

New antidepressant drugs are needed to improve the health and quality of life of the hundreds of millions of people worldwide who still suffer from depression. Over the past $10-15$ years, a growing number of studies are being conducted for the depression-based drugs and treatments that have been proven to treat 90 percent of the patients and have helped countless people with mental health problems overcome their difficulties [4]. Hundreds of articles have been published, and treatments have focused on medication and psychotherapy. But with the development of science and technology, more new treatment modalities such as non-invasive magnetic therapy [5-6] or invasive optogenetic therapy [7] are under research. To help shorten the review time and avoid duplication, it is necessary to summarize the previous mainstream treatments.

In this study, we first discuss the background and symptoms of depression, then outlines some of the major treatments currently available. Most of the known therapies in the experimental and clinical settings will be explained, and research on the mainstream treatments will be reviewed in detail. We will also propose and discuss potential therapies under investigation in the future preclinical based on available information.

\section{Overview of the Depression}

The depressive mood is a symptom of some mood disorders, such as major depression or dysthymia [8]. But in severe cases, it can rise to a mental illness. The

\footnotetext{
*Corresponding author: guanghua.ren@gecacademy.cn
} 
symptoms of depression can manifest in many ways. Such as anger, irritability, anxiety, restlessness. Or feeling empty, sad, hopeless. In severe cases, the symptoms may affect cognition or sleep and seriously impair bodily functions [9]. It can be short term or long term.

\subsection{Environmental factors lead to depression}

There are many possible causes of depression, but depression due to life experiences is probably one of the most common. Life events and changes that may affect depression include, but are not limited to: childbirth, financial hardship, social stress, medical diagnosis, bullying, loss of loved ones, natural disasters, social isolation, etc. The COVID-19 outbreak in 2020 has led to a sharp increase in people suffering from depression. A University of Surrey trial reported that the level of clinical depression among those surveyed had more than doubled, from 14.9 percent in autumn 2019 to 34.7 percent in May/June 2020 [10]. Alcohol may be an underlying cause. An Internet-based study has found that college students who are depressed and who drink alcohol have a higher risk of developing alcohol problems [11]. Depression can also be a result of medication. Isotretinoin, Rimonabant, and alphainterferons appear to pose the highest risk [12].

\subsection{Biological factors lead to depression}

Scientific studies have found that different brain regions in people with depression show different changes in activity, encouraging advocates of theories that seek to identify the biochemical source of the disease rather than theories that emphasize psychological or situational causes. Researchers have suggested that having too little certain substances in the brain, called neurotransmitters, may contribute to depression. Restoring the balance of these substances in the brain can help relieve symptoms - which is where the different classes of antidepressants available can help [13]. Neurotransmitters are chemical messengers used by the nervous system to regulate countless body functions and processes, from sleep to metabolism. The main hypothesis in the current study is that monoamines, neurotransmitters that include serotonin, dopamine, norepinephrine, and epinephrine, are responsible for depressive symptoms [14].

\section{Drug Treatments}

\subsection{Selective serotonin reuptake inhibitors (SSRIS)}

The most common way to treat depression is antidepressant drugs. And according to a survey of prescribing practices in the treatment of depression, $49 \%$ of clinicians who participate in the survey think one antidepressant drug is more efficacious than others, $48 \%$ of those people reckons that SSRIs, or selective serotonin reuptake inhibitor drugs, are most efficacious [15]. As a result, this part will introduce SSRIs, one of the first-line anti-depressant drugs, due to their effectiveness to $60 \%-80 \%$ of patients who have depression.

Firstly, the SSRIs works by inhibiting the serotonin reuptake process. Serotonin is a kind of monoamine neurotransmitter that participates in the control of mood. By increasing the level of serotonin within the brain, SSRIs relieve depression. This is because depression results from reduced activity of serotonin and norepinephrine. Interfering the reuptake process, serotonin is released and reabsorbed by the cell, SSRIs provided more serotonin available for brain activity. The mechanism is that SSRIs will block the serotonin reabsorption sites on the surfaces of nerve cells, so serotonin cannot be used again for cells. As a result, serotonin activity increased. Figure 1 shows how SSRIs works for treating Depression.

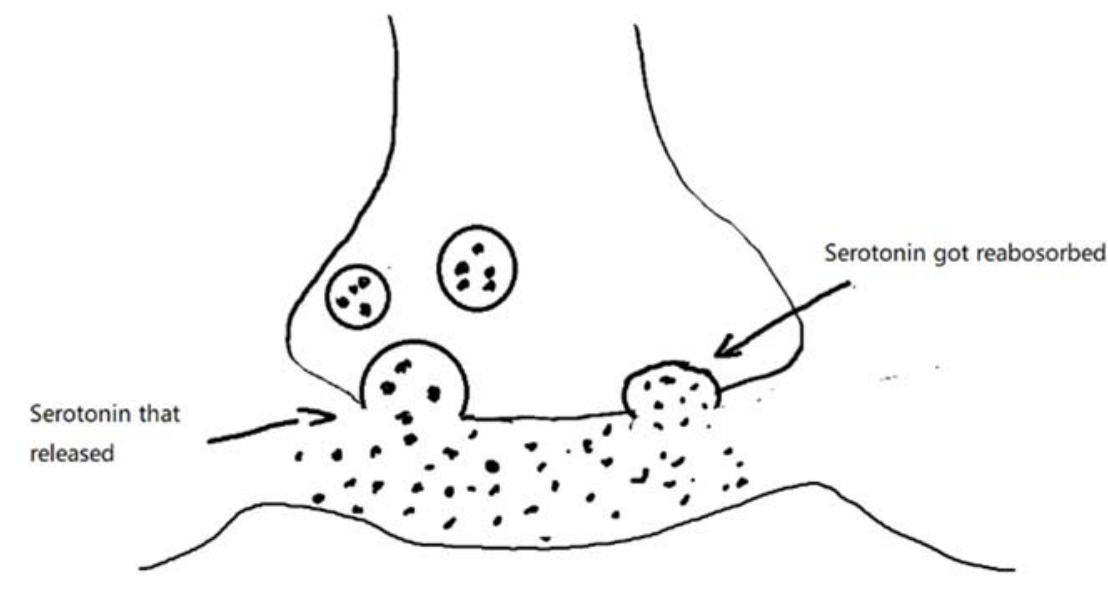

Process of serotonin reuptake 


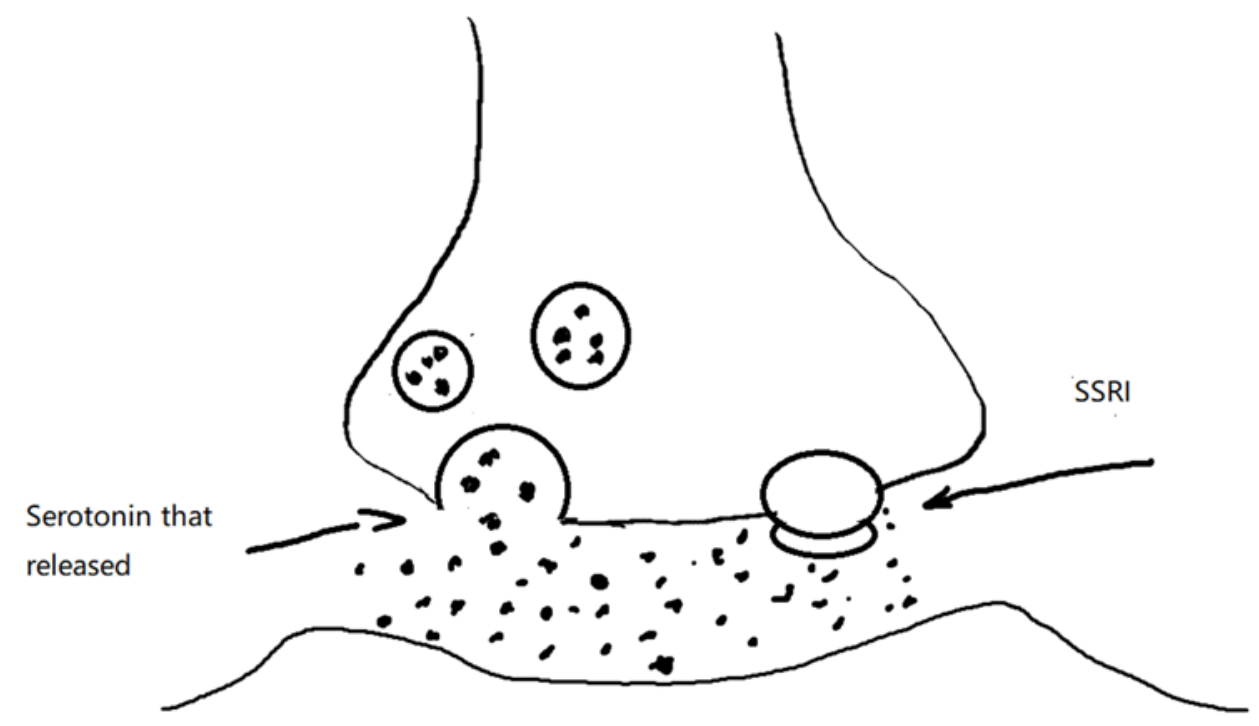

Fig. 1. Mechanism of SSRIs.

The figure on the top shows the process of serotonin reuptake. Serotonin released by the cell is reabsorbed through reabsorption sites. On the bottom figure, SSRIs increase the available serotonin by blocking the serotonin reabsorption sites, and as a result, the serotonin released will not be reused.

B R Godlewska's experiment exposes that the SSRIs are effective in the human model. After 6 weeks of treatment of SSRIs, 22 out of 35 patients were responders, people who respond to SSRIs. They showed at least a $50 \%$ reduction in symptoms and had a greater improvement in HAM-D, a method to measure depression, proving that SSRIs' effectiveness to Depression [16]. Compared with the human model, Connie Sanchez set up a mice experiment to examine whether SSRIs work to treat depression. By forcing mice to swim, mice express depression when they are immobile in the water. With treatment of Citalopram, a kind of SSRIs, mice's immobility was reversed. Another group that took Paroxetine, another kind of SSRIs, showed a 40\%-50\% reversal. Other mice depression models that were caused by foot-sock also reflected a positive result. Both journals on depression reveal the effectiveness of SSRIS [17].

Comparing with other antidepressant drugs, SSRIs are not dangerous even in overdoses. About its disadvantages, like most frugs, SSRIs also have side effects: dry mouth, dizziness, heartburn, decreased appetite, and so on. In addition, similar to many drugs, withdrawal symptoms occur in SSRIS treatment. The discontinuation syndrome maybe a runny nose, nausea, sweating, muscle pain, etc. Moreover, there are some worries about the relationship between suicide and SSRIs treatment. However, to Arif Khan's words, their experiment shows that the suicide rate in the SSRI group is similar to the placebo group. As a result, there is no connection between using SSRIs and increasing suicide rates [18]. And one important thing is that SSRIs interact with lots of drugs, like the interaction between SSRIs and MAOIs that is fatal.

\subsection{Serotonin and norepinephrine reuptake inhibitors (SNRIs)}

Desvenlafaxine (DVS) is used to treat Major Depressive Disorder (MDD). DVS is an SNRI that can block serotonin and norepinephrine reuptake and is considered a first-line agent for most types of neuropathic pain. These drugs are effective in both depressed and nondepressed patients, and the dose required to treat neuropathic pain is much lower than the dose required to treat antidepressants. The effects are usually seen within a few days rather than the weeks it takes for an antidepressant response. DVS is also being studied to treat pain [19]. The pain scale was developed as part of a large multi-center trial that determined the efficacy of a distributed pain scale in treating major depression [20]. According to Lieberman DZ, the overall VAS-PI score was significantly lower in those who received a placebo than those who received DVS. DVS provides clinicians with a limited choice for more than one neurotransmitter. It avoids the post-synaptic receptor interactions associated with the high burden of side effects of previous generations of antidepressants.

\subsection{Monoamine oxidase inhibitors (MAOls)}

MAOIs were the first class of antidepressants to be developed. The use of MAOIs usually requires dietary restriction and the avoidance of certain other medications. This antidepressant works by affecting neurotransmitters between brain cells to ease depression [21]. MAOIs remove the neurotransmitters norepinephrine, serotonin, and dopamine from the brain by blocking an enzyme called monoamine oxidase. This allows more brain chemicals to affect changes in cells and circuits affected by depression. MAOIs also affect other neurotransmitters in the brain and digestive system, leading to side effects. In addition, Isocarboxazid (Marplan) is a MAOIS drug used for the treatment of 
depression. It elevates levels of norepinephrine, serotonin, and dopamine.

\section{Other Treatments for Depression and their Mechanism}

\subsection{Psychological therapy}

The main idea for psychology is healing people's depression by communicating. Since our humans have a social relationship system and serotonin in our body will react to the stimuli, this therapy can have a warm, longterm influence on depression.

\subsubsection{Cognitive behaviour therapy (CBT)}

CBT is an effective treatment that came from the idea of the negative thinking that always led to patients themselves as the reason for depression feeling or action. It combines cognitive therapy and behavioral therapy. There is a structured set of this treatment build by the different points for two therapies [22]. While cognitive therapy focuses on changing how patients think or produced opinions to limit their condemnation of themselves, behavioral therapy led them to be more active by participating in positive activity. So, CBT can activate the reward system of patients to a certain extent.

\subsubsection{Interpersonal therapy (IPT)}

IPT's purpose is to increase the network relationship and social ability of patients. It achieved this purpose by four processes, so it is a structured psychological therapy. First, it increases the quality and insufficient of relationships. Second, it decreased the influence of resources for depression if there is a main origin cause abnormal depression. Third, it gives a smoother buffer period for patients to a life transition. Fourth, reduced injure from conflict between people's close relationship with the patient [23].

\subsubsection{Online Psychological Interventions}

By a previous study, depression often caused by shame, and one of the major feelings that patients will have is ashamed themselves [24]. This symptom will lead to depression patient hardly asked the doctor in person. Then online interventions will give them a better way. It provides a more confident area for the less extra stimulus than the patients' current tolerance.

But online psychological interventions could easily lead to ineffective by low rates of adherence. Since psychological therapy needs patient to follow the thinking habit of positive logic, and a complete goal frequently achieves it by goal, patients with fewer practices will have less effect [25].

\subsection{Electroconvulsive therapy (ECT)}

ECT is only used when patients have already finished their first treatment process but don't get valuable results. In previous years, electroconvulsive therapy has side effects greater than actual effects and gives patients pain more than its effect. By producing new technology for the anesthesia process and more controlled current, the side effect for ECT decreases to a satisfactory level by more safety protection.

ECT is a way to use electron devices to give outside stimulation to neurotransmitters. It is valuable because low current stimulation is similar to the common action potential produced by our system. Because the electron device needs to simulate the whole area of the brain, which means it cannot control the area by large precision. Nowadays, there is a new technology named Optogenetics that is testing depression to give a better option than ECT [26-27].

\subsection{Transcranial magnetic stimulation (TMS)}

TMS is another way to stimulate the brain to produce or activate more neurotransmitters. Although the principle of using magnetic fields to stimulate the navel cells is still non be found, the value of TMS can already be proven through the test on animals and actual work on humans. Since it is a non-invasive tool and does not need anesthesia, it has been seen as a safer method for depression that can be used times repetitively.

By fig. 2, when the publication about the safety of TMS increased in a range of years then decrease, it implies that the research and clinical study about TMS already reach the height that close to the highest possible safety nowadays. And high citation rate and a total number of articles in this field can show the effectiveness of TMS. This research can prove that TMS is a safe method with a high effect on depression [28].

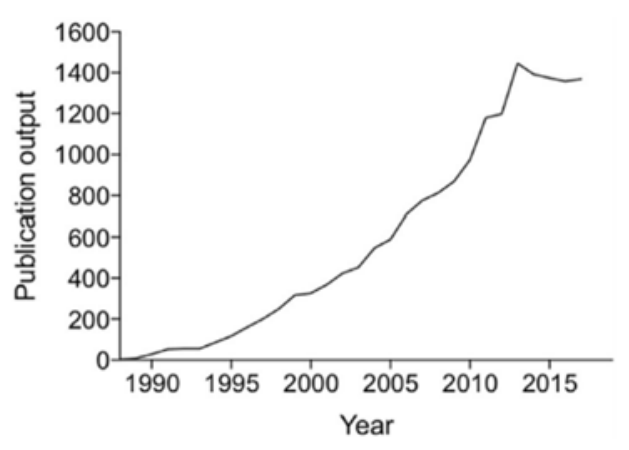

Fig. 2. Publication output per the year 1988-2017 [29].

The figure shows that the mean year-on-year growth for publication about TMS during 1988-2017 decreased every five years, and there is downhill after 2013.

\subsection{Vagus nerve stimulation (VNS)}

Vagus nerve stimulation is the long-term use of ECT on a designated location for a longer effect. Since it is an 
invasive procedure, it needs to be put inside your body by surgery and easily affect the organs around its place. Although it has a relatively larger side effect, it is useful for patients inactivated with medications and other therapy.

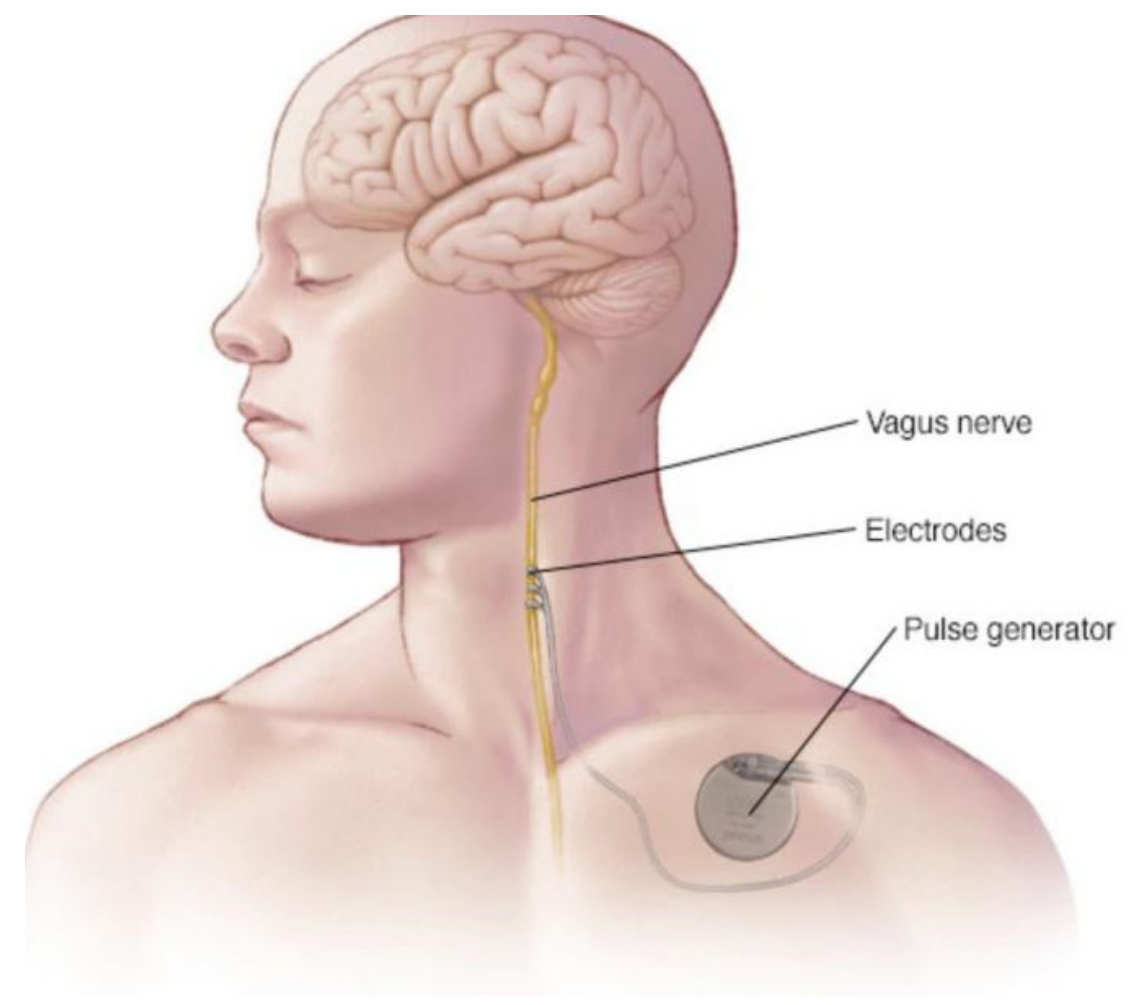

Fig. 3. Vagus nerve.

The figure shows that the way for the circuit for VNS connect with the brain with the vagus nerve and the structure of VNS. Since the circuit does not actually touch the brain, VNS has been seen as a relatively safe treatment for depression.

\section{Future Prediction}

\subsection{The development of new drugs}

Now, one drug that researchers are exploring is ketamine [30]. The drug was originally used as an anesthetic, but it is now used to treat depression. Researchers are learning more about how ketamine works so they can develop safer and more effective treatments. Studies have shown that using drugs such as ketamine may improve depression more quickly. One possible target of ketamine is the NMDA receptor in the brain. By binding to these receptors, it can increase the number of glutamate neurotransmitters between neurons. Glutamate activates connections to AMPA receptors. In general, the initial blocking of NMDA receptors and activation of AMPA receptors leads to the release of molecules that help neurons communicate through new pathways. Synaptogenesis, which affects thought patterns, mood, and cognition.

\subsection{Challenge of applying optogenetics to human}

A technique that has only been around for a few years has also been found to have the potential for treating depression. This technique is called optogenetics. The most important challenge for optogenetic is that the safety of optogenetic. To the words of Adamczyk, almost all available treatments for neurological or psychiatric disorders entail some risks and side effects [31]. Before having an accurate prediction on the adverse consequences of optogenetic, this technology will not be applied to the human brain. Another challenge is that the duration and consequence of the expression of light-sensitive proteins in the human brain are unknown [31]. Optogenetics only works when the expression of the key protein is at least endurable enough to give a long-term treatment result. Moreover, the tools now used for optogenetics are suitable for mice but not for the brain. And the last challenge is that human brain functions are not working just in a specific area. For example, the episodic memory system is related to only the hippocampus and other structures like the perirhinal, entorhinal, parahippocampal cortices, etc. [31]. As a result, applying optogenetics must considering targeting neurons of the whole network. All in all, applying optogenetics to the human need to overcome the technical issues about safety and tools, neuroscientists need to figure out how to target whole network neurons in the human brain. 


\section{Conclusion}

Due to the prevalence of depression in the country and the difficulty of intervening with its causes, the treatment of depression and the intervention of these symptoms are in demand urgently. Several commonly used drug therapies based on the principle of maintaining the balance of neurotransmitter levels are introduced, including the SSRIs, SNRIs, and MAOIs, which are widely considered to be the most effective antidepressants. These drugs work mainly by interfering with one or more neurotransmitters that may contribute to depression. At the same time, a new drug called ketamine is on research. And we have discovered a very new technique called optogenetics, which is worth studying for treating depression. However, due to the limitations of technology and the human brain structure, a long period of research is necessary before this technique can be successfully applied to the human brain. More analysis and research of the human brain and related animal models should be performed to improve the success rate. Further research is still necessary before more treatments are introduced to the public.

\section{References}

1. $\mathrm{Hu} \mathrm{L}$, Liang J, Jin SY, Han YJ, Lu J, Tu Y. [Progress of researches on mechanisms of acupuncture underlying improvement of depression in the past five years]. Zhen Ci Yan Jiu. 2013 Jun;38(3):253-8. Chinese.

2. Glover GR. Unrecognised depression in general practice. Br Med J (Clin Res Ed). 1985 Jul 13;291(6488): 140 .

3. de Zwart, P. L., Jeronimus, B. F., \& de Jonge, P. (2019). Empirical evidence for definitions of episode, remission, recovery, relapse and recurrence in depression: a systematic review. Epidemiology and psychiatric sciences, 28(5), 544-562.

4. Cole, E. J., Stimpson, K. H., Bentzley, B. S., Gulser, M., Cherian, K., Tischler, C., Nejad, R., Pankow, H., Choi, E., Aaron, H., Espil, F. M., Pannu, J., Xiao, X., Duvio, D., Solvason, H. B., Hawkins, J., Guerra, A., Jo, B., Raj, K. S., Phillips, A. L., '. Williams, N. R. (2020). Stanford Accelerated Intelligent Neuromodulation Therapy for Treatment-Resistant Depression. The American journal of psychiatry, 177(8), 716-726.

5. George, M. S., Wassermann, E. M., Williams, W. A., Callahan, A., Ketter, T. A., Basser, P., Hallett, M., \& Post, R. M. (1995). Daily repetitive transcranial magnetic stimulation (rTMS) improves mood in depression. Neuroreport, 6(14), 1853-1856.

6. Pascual-Leone A, Rubio B, Pallardó F, Catalá MD. Rapid-rate transcranial magnetic stimulation of left dorsolateral prefrontal cortex in drug-resistant depression. Lancet. 1996 Jul 27;348(9022):233-7.

7. Battle DE. Diagnostic and Statistical Manual of Mental Disorders (DSM). Codas. 2013;25(2):191-2.
8. Hirschfeld RM. Major depression, dysthymia and depressive personality disorder. Br J Psychiatry Suppl. 1994 Dec;(26):23-30.

9. Albert PR. Light up your life: optogenetics for depression? J Psychiatry Neurosci. 2014 Jan;39(1):3-5.

10. Evans S, Alkan E, Bhangoo JK, Tenenbaum H, NgKnight T. Effects of the COVID-19 lockdown on mental health, wellbeing, sleep, and alcohol use in a UK student sample. Psychiatry Res. 2021 Apr;298:113819.

11. Rogers D, Pies R. General medical with depression drugs associated. Psychiatry (Edgmont). 2008 Dec;5(12):28-41.

12. Geisner IM, Mallett K, Varvil-Weld L, Ackerman S, Trager BM, Turrisi R. An examination of heavy drinking, depressed mood, drinking related constructs, and consequences among high-risk college students using a person-centered approach. Addict Behav. 2018 Mar;78:22-29.

13. Nutt DJ. Relationship of neurotransmitters to the symptoms of major depressive disorder. J Clin Psychiatry. 2008;69 Suppl E1:4-7.

14. Hirschfeld RM. History and evolution of the monoamine hypothesis of depression. J Clin Psychiatry. 2000;61 Suppl 6:4-6.

15. Petersen T, Dording C, Neault NB, Kornbluh R, Alpert JE, Nierenberg AA, Rosenbaum JF, Fava M. A survey of prescribing practices in the treatment of depression. Prog Neuropsychopharmacol Biol Psychiatry. 2002 Jan;26(1):177-87.

16. Godlewska BR, Browning M, Norbury R, Cowen PJ, Harmer CJ. Early changes in emotional processing as a marker of clinical response to SSRI treatment in depression. Transl Psychiatry. 2016 Nov 22;6(11):e957.

17. Sánchez C, Meier E. Behavioral profiles of SSRIs in animal models of depression, anxiety and aggression. Are they all alike? Psychopharmacology (Berl). 1997 Feb;129(3):197-205.

18. Khan A, Khan S, Kolts R, Brown WA. Suicide rates in clinical trials of SSRIs, other antidepressants, and placebo: analysis of FDA reports. Am J Psychiatry. 2003 Apr;160(4):790-2.

19. Carlsson AM. Assessment of chronic pain. I. Aspects of the reliability and validity of the visual analogue scale. Pain. 1983 May;16(1):87-101.

20. Lieberman DZ, Montgomery SA, Tourian KA, Brisard C, Rosas G, Padmanabhan K, Germain JM, Pitrosky B. A pooled analysis of two placebocontrolled trials of desvenlafaxine in major depressive disorder. Int Clin Psychopharmacol. 2008 Jul;23(4):188-97.

21. Youdim MB, Edmondson D, Tipton KF. The therapeutic potential of monoamine oxidase inhibitors. Nat Rev Neurosci. 2006 Apr;7(4):295309. 
22. Zhang A, Borhneimer LA, Weaver A, Franklin C, Hai AH, Guz S, Shen L. Cognitive behavioral therapy for primary care depression and anxiety: a secondary meta-analytic review using robust variance estimation in meta-regression. J Behav Med. 2019 Dec;42(6):1117-1141.

23. Weissman MM. Interpersonal Psychotherapy: History and Future. Am J Psychother. 2020 Mar 1;73(1):3-7.

24. Scheff TJ. Shame and community: social components in depression. Psychiatry. 2001 Fall;64(3):212-24.

25. Beatty L, Binnion C. A Systematic Review of Predictors of, and Reasons for, Adherence to Online Psychological Interventions. Int J Behav Med. 2016 Dec;23(6):776-794.

26. Lobo MK, Nestler EJ, Covington HE 3rd. Potential utility of optogenetics in the study of depression. Biol Psychiatry. 2012 Jun 15;71(12):1068-74. doi: 10.1016/j.biopsych.2011.12.026. Epub 2012 Feb 8. PMID: 22322104; PMCID: PMC3738208.

27. Golden SA, Covington HE 3rd, Berton O, Russo SJ. A standardized protocol for repeated social defeat stress in mice. Nat Protoc. 2011 Jul 21;6(8):1183-91.

28. Rossi S, Hallett M, Rossini PM, Pascual-Leone A; Safety of TMS Consensus Group. Safety, ethical considerations, and application guidelines for the use of transcranial magnetic stimulation in clinical practice and research. Clin Neurophysiol. 2009 Dec;120(12):2008-2039.

29. Lawson McLean A. Publication trends in transcranial magnetic stimulation: a 30-year panorama. Brain Stimul. 2019 May-Jun;12(3):619627.

30. Averill, Lynnette A et al. "Chronic stress pathology and ketamine-induced alterations in functional connectivity in major depressive disorder: An abridged review of the clinical evidence." Advances in pharmacology (San Diego, Calif.) vol. 89 (2020): 163-194.

31. Adamczyk, A.K., Zawadzki, P. The MemoryModifying Potential of Optogenetics and the Need for Neuroethics. Nanoethics 14, 207-225 (2020). 